\title{
Ground-State Electron Transfer as an Initiation Mechanism for Asymmetric Hydroalkylations in Radical Biocatalysis
}

Haigen Fu, ${ }^{1,2}$ Heather Lam, ${ }^{1,2}$ Megan A. Emmanuel, ${ }^{1}$ Ji Hye Kim, ${ }^{1}$ Braddock A. Sandoval, ${ }^{1}$ Todd K. Hyster ${ }^{1,2 *}$

Affiliation:

${ }^{1}$ Department of Chemistry, Princeton University, Princeton, New Jersey 08544, United States

${ }^{2}$ Department of Chemistry and Chemical Biology, Cornell University, Ithaca, NY 14853, United States

*Correspondence to: thyster@cornell.edu

\begin{abstract}
Stereoselective bond-forming reactions are essential tools in modern organic synthesis. However, catalytic strategies for controlling the stereochemical outcome of radicalmediated $\mathrm{C}-\mathrm{C}$ bond formation remain underdeveloped. Here, we report an 'ene'reductase catalyzed asymmetric hydroalkylation of olefins using $\alpha$-bromoketones as radical precursors. In these reactions, radical initiation occurs via ground-state electron transfer from the flavin cofactor located within the enzyme active site, representing a mechanistic departure from previous photoenzymatic hydroalkylations. Four rounds of site saturation mutagenesis based on wild-type nicotinamide-dependent cyclohexanone reductase (NCR) were deployed to access a variant capable of catalyzing a cyclization to furnish $\beta$-chiral cyclopentanones with high levels of enantioselectivity. Additionally, the wild-type NCR was identified that could catalyze the intermolecular coupling with precise stereochemical control over the radical termination step. This report demonstrates this enzyme family's catalytic versatility and highlights the opportunity for protein engineering to address reactivity and selectivity challenges in radical biocatalysis.
\end{abstract}




\section{Introduction}

Radicals are versatile synthetic intermediates that enable numerous fascinating processes and retrosynthetic disconnections towards complex molecules. ${ }^{1-3}$ While the overall reactivity of radical intermediates is well understood, ${ }^{4}$ catalytic strategies for controlling the chemo- and stereoselectivity remain underdeveloped. ${ }^{5-7}$ Nature has evolved various biocatalysts, such as cobalamin and radical $S$-adenosylmethionine (SAM) enzymes, to catalyze the radical-mediated transformations with unparalleled chemo-, regio-, and enantioselectivity in the biosynthesis of complex natural products. ${ }^{8-12}$ However, these enzymes are not readily harnessed outside of their natural settings by organic synthetic chemists, owing to either operational difficulties or lack of catalytic versatility. We imagined that enzymes could be used more broadly to solve selectivity challenges in the radical literature by identifying and developing enzymes that can use non-natural mechanisms for radical formation. ${ }^{13,14}$

Flavin-dependent 'ene'-reductases (EREDs) naturally catalyze the asymmetric reduction of electronically activated alkenes and are among the most widely used class of biocatalysts in chemical synthesis. ${ }^{15,16}$ Mechanistically, the reaction occurs via a stereoselective hydride transfer from the cofactor flavin hydroquinone $\left(\mathrm{FMN}_{\mathrm{hq}}\right)$ to the substrate's electrophilic position (Fig. 1a) followed by protonation by a conserved tyrosine residue in the enzyme active-site to give the hydrogenated product. ${ }^{15,16}$ Beyond its canonical ionic mechanism, $\mathrm{FMN}_{\mathrm{hq}}$ can also serve as a single-electron reductant $\left(\mathrm{E}_{1 / 2}\right.$ $=-450 \mathrm{mV}$ versus saturated calomel electrode) (Fig. 1a). ${ }^{17,18}$ In our previous study, we found that EREDs can catalyze an asymmetric radical hydrodehalogenation of $\alpha$-bromo esters, giving rise to enantioenriched ester products (Fig. 1a, Bottom). ${ }^{19}$ In this reaction, radical formation occurs via a ground-state electron transfer from $\mathrm{FMN}_{\mathrm{hq}}$ to the $\alpha$-bromo ester, forming an $\alpha$-acyl radical and the partially oxidized flavin semiquinone ( $\left.F M N_{\mathrm{sq}}\right)$ (Fig. 1a, Bottom). ${ }^{19}$ Interestingly, this mechanism can accommodate modestly endergonic electron transfers, making it ideal for $\alpha$-bromoesters and ketones while also avoiding the need for more complicated photoinduced electron transfer mechanisms that are required for less oxidizing substrates. ${ }^{20-22}$ Inspired by the simplicity of this 
initiation mechanism, we questioned whether it could be used for the asymmetric hydroalkylation of olefins, a challenging transformation to render asymmetric using small molecule catalysts. ${ }^{6,7}$

As a model, we targeted the cyclization of $\alpha$-bromoketones 1 to afford $\beta$ stereogenic cyclopentanone $\mathbf{2}$ (Fig. 1b). The cyclopentanone motif is prevalent in natural products and biologically active molecules, such as Prostaglandin E1 (PGE1, 6), ${ }^{23}$ plant hormone Jasmonic acid $7,{ }^{24}$ terpenoid $\beta$-Cuparenone $8,{ }^{25}$ antibacterial agent Sarkomycin 9, ${ }^{26}$ and malarial aspartic proteases inhibitor $\mathbf{1 0}$ (Fig. 1c). ${ }^{27}$ We envisioned a reaction mechanism where (i) ground-state electron transfer from $\mathrm{FMN}_{\mathrm{hq}}$ to the $\alpha$ bromoketone 1 would afford $\alpha$-acyl radical 4, (ii) cyclization of this intermediate to produce a new carbon-centered radical 5, and (iii) radical termination via HAT from $\mathrm{FMN}_{\mathrm{sq}}$ to afford the desired product (Fig. 1b). We anticipated the intermediacy of $\alpha$ ketonyl radicals would present distinct challenges by comparison to our previous biocatalytic cyclization involving $\alpha$-amidyl radicals. ${ }^{20}$ Specifically, $\alpha$-ketonyl radicals have lower reduction potentials and are more reactive, making them prone to undesired electron and hydrogen atom transfer pathways that ultimately form undesired hydrodehalogenated products. ${ }^{28}$ These side reactions also plague variants of this reaction mediated by small-molecule reagents. ${ }^{29-31}$ The enhanced reactivity of this intermediate should also result in a transition state for the $\mathrm{C}-\mathrm{C}$ bond-forming step that more closely resembles the starting material (per the Hammond Postulate), making it harder to achieve high levels of enantioselectivity, potentially accounting for the lack of general small molecules catalysts for this reaction (Fig. 1b). ${ }^{32}$ 

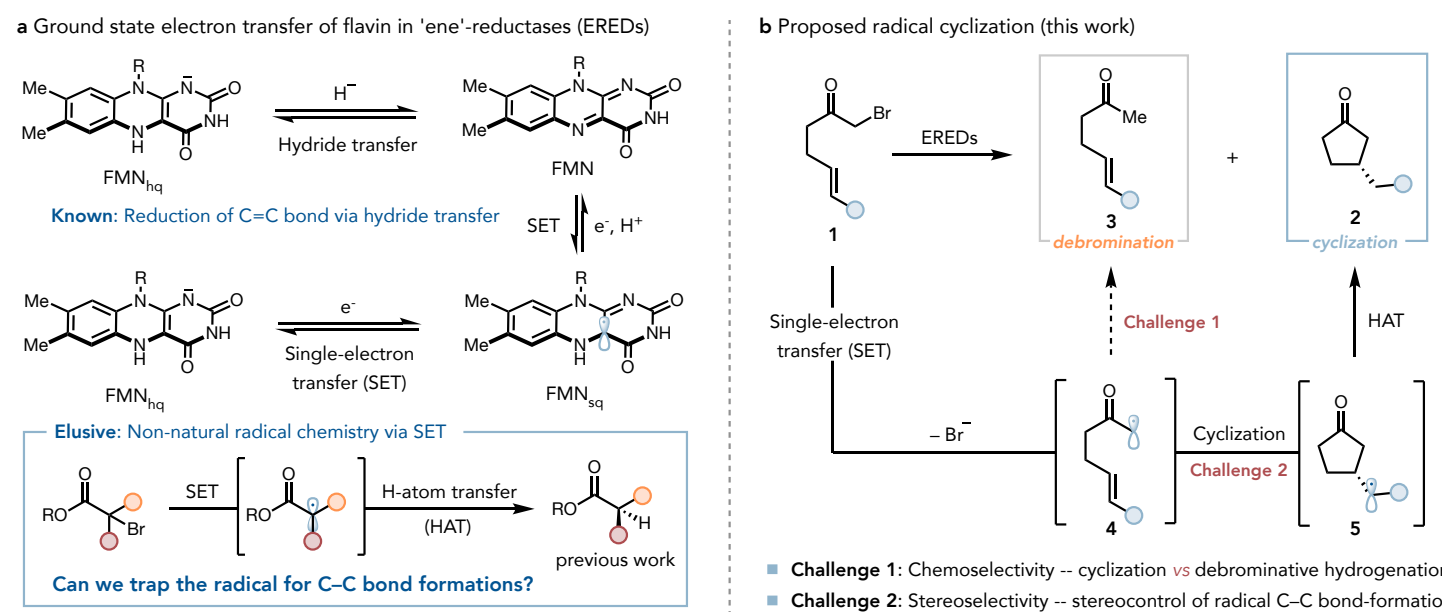

an Challenge 1: Chemoselectivity -- cyclization vs debrominative hydrogenation

- Challenge 2: Stereoselectivity -- stereocontrol of radical C-C bond-formation

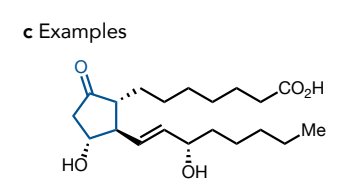

6

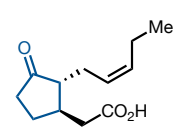

7

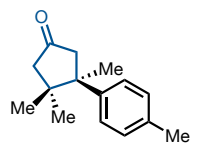

8

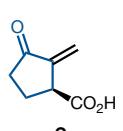

9

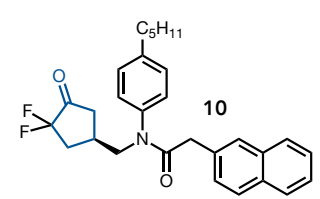

Fig. 1. Strategies and challenges in using 'ene'-reductases (EREDs) for nonnatural radical cyclization. a, The viable ground-state electron transfer of flavin in EREDs. Hydride transfer is well documented in the asymmetric reduction of electronically activated $\mathrm{C}=\mathrm{C}$ double bonds by EREDs. In contrast, non-natural radical chemistry involving ground-state single-electron transfer (SET) is elusive. b, The proposed asymmetric radical cyclization involving ground-state SET using EREDs and its associated two challenges: controlling chemo- and stereoselectivity. c, Natural products and biologically active molecules containing the cyclopentanone core structure.

\section{Results}

\section{Discovery and engineering of NCR for radical cyclization}

We began our studies by exploring the reductive cyclization of $\alpha$-bromoketone 1a to $\beta$-stereogenic cyclopentanone $\mathbf{2 a}$ catalyzed by structurally diverse wild-type EREDs under ambient conditions with an NADPH regeneration system (GDH/NADP ${ }^{+} /$glucose) (Supplementary Table 1). Several EREDs provided the desired cyclopentanone product 2a, although significant quantities of the hydrodehalogenation product $\mathbf{3}$ were observed in all cases. Control experiments confirmed that both ERED and the NADPH regeneration system were required for reactivity. The radical cyclization catalyzed by nicotinamide-dependent cyclohexanone reductase (NCR) from Zymomonas mobilis 
afforded product $\mathbf{2 a}$ in $20 \%$ yield and moderate enantioselectivity (79:21 er) with a significant amount of the dehalogenated product 3 (19\% yield) and the remaining balance being starting material 1a. The ERED 12-oxophytodienoate reductase 1 (OPR1) from Solanum lycopersicum provided a slightly improved yield and enantioselectivity (31\% yield, 80:20 er) for product 2a, with less side product 3 (9\% yield, Supplementary Table 1). Importantly, this reactivity is also found in commercial ERED collections. For instance, ERED-30 from the Prozomix library affords product 2a in 94\% yield (65:35 e.r.) and only $6 \%$ yield of the linear dehalogenated product 3 (Supplementary Table 1). As a wild-type enzyme could not be identified that affords high yields and enantioselectivities; we chose to evolve an improved catalyst.

Wild-type NCR was selected as the starting template because of its superior expression profile by comparison to OPR1. Evolution was conducted using iterative rounds of site-saturation mutagenesis (SSM). Amino acid residues chosen for SSM included those that line the active site pocket and interact with either the substrate or flavin cofactor (the docking model of substrate 1a in wild-type NCR is shown in Supplementary Fig. 1). ${ }^{33}$ For each round of SSM, enzyme libraries were co-expressed with the groES-groEL chaperone using Escherichia coli cells and screened in 96-well plates in the form of cell-free extracts (CFEs). After four rounds of SSM, we discovered four beneficial mutations Y343W, F269W, W342A, and I231S, which collectively furnishing a notable 7.7-fold improvement in yield and further enhancements in enantioselectivity; the undesired dehalogenated linear product 3 was also effectively diminished to a negligible level (Fig. 2 and Supplementary Tables 2-10). Using the final quadruple mutant (Y343W/F269W/W342A/I231S), named NCR-C9, as the biocatalyst under optimized conditions, the $(S)$-configured cyclization product 2 a was formed in 92\% yield and 95:5 er, with a 99:1 ratio of cyclized/linear (2a/3) products (Fig. 2). This reaction can be run on a preparative scale and afford product in $86 \%$ isolated yield with no changes in enantio- or chemoselectivity (Scheme 1). 
With the evolved non-natural radical enzyme NCR-C9 in hand, we explored the transformation scope and limitations (Scheme 1). The quadruple mutant NCR-C9 accommodated various para-substituents of the aromatic ring, with electron-donating (1e-f) and electron-withdrawing (1i) substituents affording $\beta$-stereogenic cyclopentanones in high yields (74-86\%) and excellent enantioselectivities ( $>94: 6 \mathrm{er}$, Scheme 1). Notably, para-halogenated substrates $(\mathbf{1 g}-\mathbf{h})$ were tolerated providing a handle for potential downstream manipulation (Scheme 1). Ortho-substituted (1b) and meta-substituted (1c-d) substrates were also well accepted by the evolved NCR-C9, giving the corresponding cyclized products $\mathbf{2 c}-\mathbf{d}$ with good yields (57-81\%) and high levels of enantioselectivity ( $>$ 90:10 er, Scheme 1). The aromatic substituent proved essential for the desired reactivity, with unsubstituted alkene or enone affording the hydrodehalogenated products exclusively (Scheme 1 and Supplementary Fig. 2).

Protein engineering campaigns result in catalysts that are better suited for the reaction on which they were evolved. To probe the specialization in this protein engineering campaign, we tested alternative substrates and cyclization modes. The (Z)isomer of the model substrate (Z)-1a was accepted by the NCR-C9, although with diminished yield and enantioselectivity (62\% yield, 69:31 er) by comparison to the $(E)$ configured substrate $(E)$-1a, indicating the importance of geometric configuration of the substrate during the NCR-catalyzed radical cyclization reaction (Scheme 1). In contrast, $(E)$-1a and (Z)-1a perform similarly with wild-type NCR, indicating that the protein engineering campaign has enhanced the preference for the $(E)$-olefin isomer. In the realm of alternative cyclization modes, the specialization was less pronounced. While NCR-C9 was ineffective for a 6-exo-trig cyclization, the wild-type enzyme could catalyze the reaction in low yield, indicating the parent enzyme is more promiscuous. In contrast, both variants afforded similar results for 7-exo-trig cyclization (Scheme 1). In addition, both the wild-type and evolved variants can catalyze the 6-endo-trig cyclization providing $\gamma$-substituted cyclohexanone $\mathbf{2 m}$ in moderate yield; however, 5endo-trig cyclization was inaccessible to both enzymes (Scheme 1 and Supplementary Fig. 3). Collectively, these results indicate that specialization is apparent within 
individual cyclization modes but does not necessarily hold when looking at other manifolds.
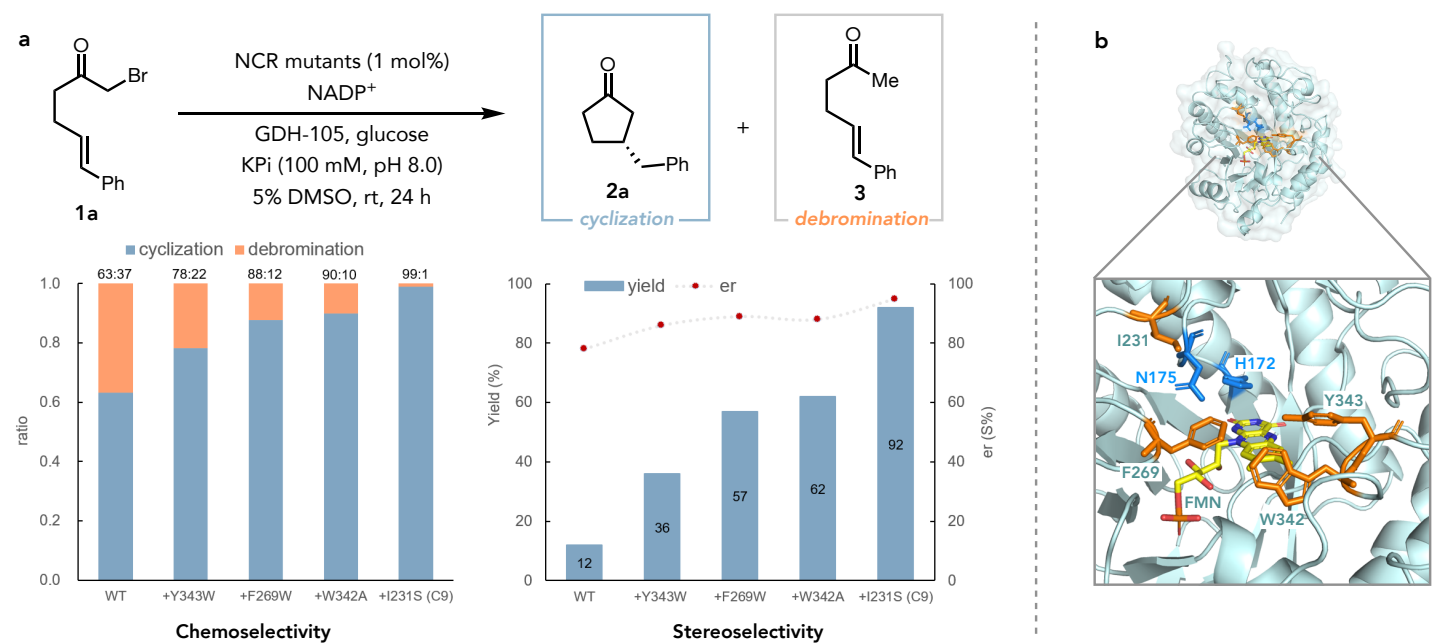

Fig. 2. Directed evolution of NCR catalysts for non-natural asymmetric radical cyclization a, Chemoselectivity (cyclization versus debrominative hydrogenation), stereoselectivity, and yields improvement of NCR variants over wild-type NCR. Standard reaction conditions: the reaction mixture $(500 \mu \mathrm{L})$ consisted of model substrate (1a, $0.004 \mathrm{mmol}), \mathrm{GDH}-105$ (0.5 mg), glucose (5.0 mg), NADP ${ }^{+}(0.5 \mathrm{mg})$, and purified NCR variants (1.0 mol\%) in buffer (100 mM KPi buffer $\mathrm{pH} 8.0)$ with $5 \%$ DMSO as cosolvent at room temperature for $24 \mathrm{~h}$. Yields were determined via HPLC relative to an internal standard (average of duplicate). Enantiomeric ratio (er) was determined via HPLC on a chiral stationary phase. b, Crystal structure of wild-type NCR (PDB: 4s3u). Residues for substrate binding (H172 and N175) are shown as blue sticks. Four beneficial sites Y343, F269, W342, and I231 are shown in orange sticks. 

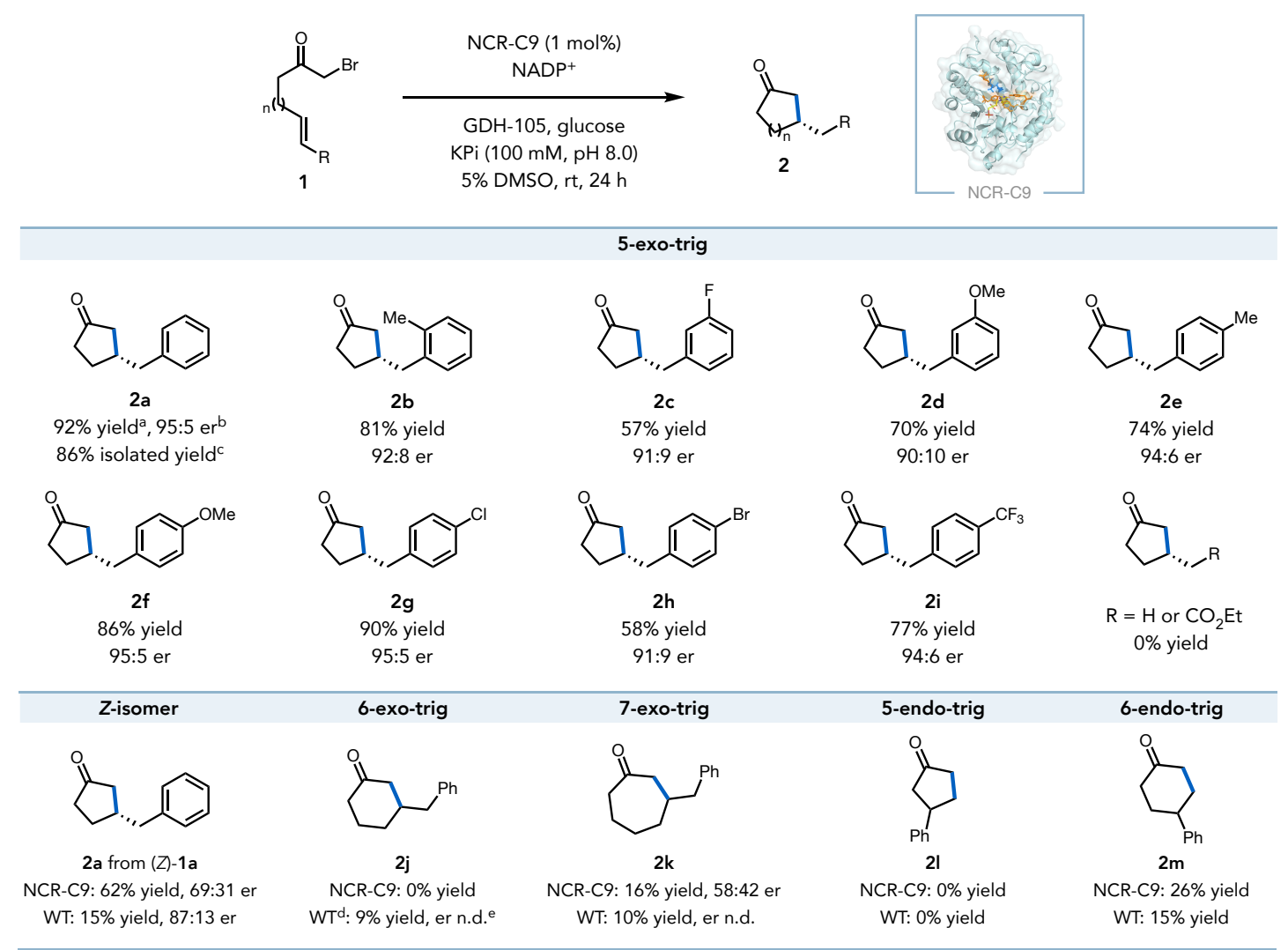

Scheme 1. Asymmetric radical cyclization catalyzed by NCR-C9 mutant. Standard reaction conditions: the reaction mixture $(500 \mu \mathrm{L})$ consisted of substrate $(\mathbf{1 a}, 0.004$ mmol), GDH-105 (0.5 mg), glucose (5.0 mg), NADP ${ }^{+}(0.5 \mathrm{mg})$, and purified NCR-C9 mutant (1.0 mol\%) in buffer (100 mM KPi buffer $\mathrm{pH} 8.0)$ with 5\% DMSO as cosolvent at room temperature for $24 \mathrm{~h} .{ }^{a}$ Yields were determined via HPLC relative to an internal standard. ${ }^{b}$ Enantiomeric ratio (er) was determined via HPLC on a chiral stationary phase. ${ }^{c}$ Isolated yield was based on 0.08 mmol-scale reaction. ${ }^{d} 2 \mathrm{~mol} \%$ of wild-type NCR was used. ${ }^{e}$ n.d., er not determined. 


\section{Intermolecular radical $\mathrm{C}-\mathrm{C}$ bond formation catalyzed by wild-type NCR}

Having evolved a selective enzyme for a radical cyclization, we further questioned whether EREDs could catalyze an intermolecular radical hydroalkylation. The central challenge in achieving such a reaction is controlling product selectivity. As both the bromoketone and alkene need to simultaneously occupy the protein active site to form the coupled product, a mechanism is required for gating electron transfer. In the absence of such a mechanism, we would expect to form the hydrodehalogenated product primarily. $^{21}$

We tested a panel of wild-type EREDs on the intermolecular hydroalkylation of $\alpha$ bromo acetophenone 11a with $\alpha$-methyl styrene 12a to provide $\gamma$-stereogenic ketone 13a (Supplementary Table 11). Several EREDs afforded the coupled product, demonstrating the feasibility of our hypothesis. Remarkably, wild-type NCR catalyzed the desired transformation in a quantitative yield (using alkene as the limiting reagent) with excellent enantioselectivity (97:3 er) for the $(R)$-enantiomer, outperforming other tested wild-type EREDs (Supplementary Table 11). The (S)-enantiomer of 13a can also be accessed using Yersina bercovieri alkene reductase (YersER) as the biocatalyst, albeit in moderate yield and enantioselectivity (44\% yield, 83:17 er). After optimizing the experimental parameters, wild-type NCR ( 1.5 mol\% loading) catalyzed the nonnatural intermolecular hydroalkylation providing the $(R)-\mathbf{1 3 a}$ in $99 \%$ yield $(82 \%$ isolated yield, Scheme 2) and excellent enantioselectivity (99:1 er). Control experiments confirmed that both NCR and the NADPH regeneration system are necessary for this biotransformation (Supplementary Table 12).

A variety of $\alpha$-bromo aryl ketones are well tolerated by the reaction (Scheme 2). $\alpha$ bromoketones possessing either electron-donating $(\mathbf{1 1 b}-\mathbf{c})$ or electron-withdrawing (11d-i) substituents at the para-position were efficiently converted to the desired enantioenriched $\gamma$-chiral ketones $(\mathbf{1 3 b}-\mathbf{i})$ in yields of $43-98 \%$ with excellent enantioselectivity ( $>$ 97:3 er). The meta-substituted substrates $\mathbf{1 1} \mathbf{j}-\mathbf{k}$ were also well accepted by the wild-type NCR, providing the corresponding products $\mathbf{1 3} \mathbf{j}-\mathbf{k}$ with good 
yields (72-96\%) and high levels of enantioselectivity (>97:3 er). Beyond acetophenone derivatives, simple $\alpha$-bromo alkyl ketone 11n was also successful in the reaction, giving rise to the product $13 \mathrm{n}$ in $63 \%$ yield and $95: 5$ er, highlighting the generality of the ground-state electron transfer mechanism. Unfortunately, $\alpha$-bromo acetic acid and $\alpha$ bromo esters were unreactive (Supplementary Fig 4). Interestingly, $\alpha$-chloro acetophenone was also reactive (49\% yield, 97:3 er), indicating that photoinduced electron transfer is not required for radical initiation (Supplemental Table 12, entry 2). ${ }^{22}$

The alkene scope is also broad for the NCR-catalyzed intermolecular hydroalkylation (Scheme 2). Various ortho- and meta-substituted $\alpha$-methyl styrenes were well accepted by the enzyme, providing the corresponding $\gamma$-chiral ketones $130-\mathbf{r}$ in yields of $58 \%-88 \%$ with excellent enantioselectivity (up to 99:1 er, Scheme 2). Parasubstituted $\alpha$-methyl styrenes were less tolerated in the reaction. The smaller para-F substituent (13s, 99\% yield, 95:5 er) outperformed larger groups such as para-Me (13t) or para-Cl (13u) substituent, which afforded only moderate yields (59-69\%) and poor enantioselectivity (Scheme 2). Beyond styrenyl alkenes, wild-type NCR could also accept heterocycles, including an electron-deficient $\alpha$-methylvinylpyridine and electron-rich $\alpha$-methylvinythiophene, providing the respective products $13 \mathbf{w}-\mathbf{x}$ in 61-74\% yield and high enantioselectivity (up to 99:1 er). This enzyme, however, was limited to small substituents at the $\alpha$-position of styrene (13y). Larger groups, such as $i$-propyl and $n$-propyl, were poorly reactive (Scheme 2 ). We hypothesize that this limitation could be overcome using protein engineering. Finally, aliphatic alkenes, such as $\alpha$-methylvinycyclohexane, were not reactive (Scheme 2 and Supplementary Fig. 4). 


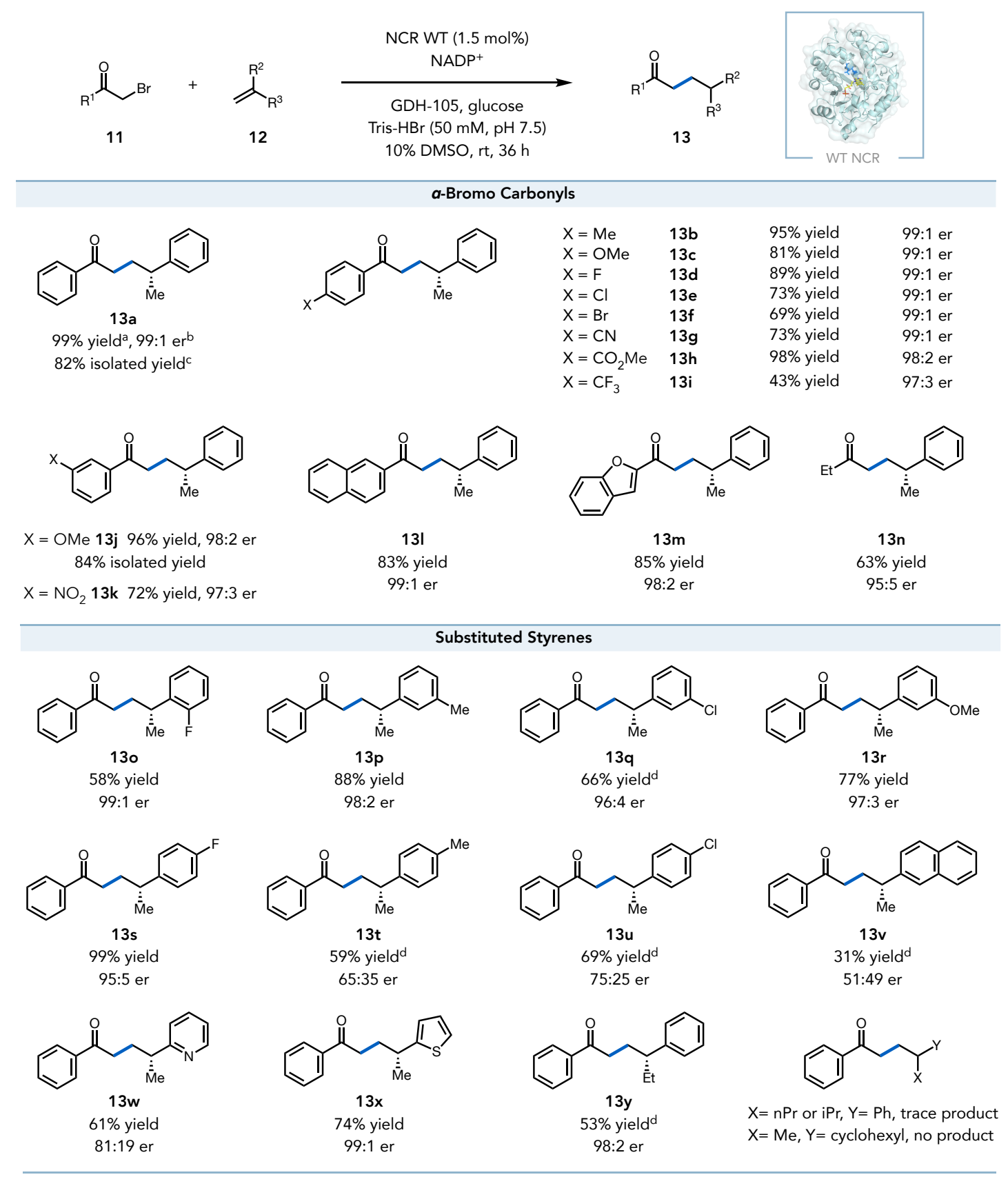

Scheme 2. Asymmetric intermolecular radical hydroalkylation catalyzed by wide-

type NCR. Standard reaction conditions: the reaction mixture $(1.2 \mathrm{~mL})$ consisted of substituted styrene (12, $0.005 \mathrm{mmol}, 1 \mathrm{eq}), \alpha$-bromo carbonyl (11, $0.015 \mathrm{mmol}, 3 \mathrm{eq})$, GDH-105 (0.3 mg), glucose (5.0 mg), NADP ${ }^{+}(0.2 \mathrm{mg})$, and purified wild-type NCR protein $(1.5 \mathrm{~mol} \%$ to alkene) in buffer (50 mM Tris-HBr buffer $\mathrm{pH} 7.5)$ with $10 \%$ DMSO as cosolvent at room temperature for $36 \mathrm{~h} .{ }^{a}$ Yields were determined via HPLC relative to an internal standard. ${ }^{b}$ Enantiomeric ratio (er) was determined via HPLC on a chiral stationary phase. ${ }^{c}$ Isolated yields were based on 0.1 mmol-scale reaction. ${ }^{d} \mathrm{An}$ 
excess of styrene $(\mathbf{1 2}, 0.015 \mathrm{mmol}, 3 \mathrm{eq})$ to $\alpha$-bromo carbonyl $(\mathbf{1 1}, 0.005 \mathrm{mmol}, 1 \mathrm{eq})$ was used.

\section{Mechanistic Experiments}

To elucidate the mechanism of termination for the benzylic radical in the NCRcatalyzed intra- and intermolecular $\mathrm{C}-\mathrm{C}$ bond-forming reactions, we conducted a set of deuterium-labeling experiments (Fig. 3 and Supplementary Fig. 6-11). For the cyclization mediated by NCR-C9, when cofactor flavin was labeled in situ using $d_{1}$-glucose and glucose dehydrogenase (GDH), the product $\beta$-benzyl cyclopentanone 2a was obtained in $83 \%$ yield and $95: 5$ er with $47 \%$ deuterium incorporation at the benzylic position (Fig. 3a). Alternatively, when the model cyclization reaction was performed in deuterated buffer, the product 2a was observed in high yield and enantioselectivity ( $93 \%$ yield, $95: 5$ er) with only $11 \%$ of benzylic deuterium incorporation (Fig. 3a). Collectively, these deuterium-labeling data suggest that the primary mechanism of quenching the benzylic radical would be HAT from flavin $\left(\mathrm{FMN}_{\mathrm{sq}}\right)$, as proposed in Fig. $1 \mathrm{~b}$ and Supplementary Fig. 5a. In the experiment where in situ deuterium-labeled flavin was used, the moderate deuterium incorporation (47 $\mathrm{D} \%$ ) of product $\mathbf{2 a}$ can be rationalized by the deuterium exchange at the flavin N5 position with $\mathrm{H}_{2} \mathrm{O}$ buffer, followed by HAT to the benzylic radical. ${ }^{34}$

For the intermolecular hydroalkylation catalyzed by wide-type NCR, when flavin was labeled in situ using $d_{1}$-glucose and GDH, the $\gamma$-chiral ketone product 13a was formed in $42 \%$ yield with 99:1 er and $80 \%$ deuterium incorporation at the benzylic position (Fig. 3b). Additionally, when this reaction was carried out in a deuterated buffer, the yield of product 13a improved to $94 \%$ (99:1 er), but hardly any deuterium incorporation at the benzylic position of 13a was observed (Fig. 3b). Collectively, these results suggest the benzylic radical is primarily terminated via HAT from flavin $\left(\mathrm{FMN}_{\mathrm{sq}}\right)$, which also set the $\gamma$-stereocenter of the hydroalkylation products (Supplementary Fig. 5b). 
Finally, we were interested in whether the presence of alkene influenced radical formation. This was determined by measuring the consumption of $\alpha$-bromo acetophenone 11a with and without $\alpha$-methylstyrene 12a. In the presence of 3 equivalents of 12a, 11a was consumed at $7.6 \mathrm{mmol} /$ hour. In the absence of alkene, that rate decreased to $3.0 \mathrm{mmol} /$ hour, which was a $2.5 \mathrm{x}$ decrease to the initial rate (Supplementary Fig. 12-14). As we expect C-Br bond cleavage to be irreversible, these data suggest that the presence of alkene impacts the formation of the $\alpha$-acyl radical, providing an empirical rationale for the high degree of product selectivity. Studies are ongoing to identify a specific molecular origin for this observation.

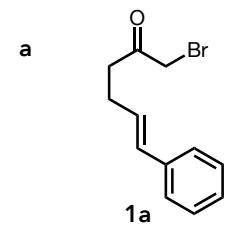

Isotopically labelled Flavin

$\mathrm{D}_{1}$-glucose, $\mathrm{H}_{2} \mathrm{O}$ buffer

Isotopically labelled buffer $\mathrm{H}_{1}$-glucose, $\mathrm{D}_{2} \mathrm{O}$ buffer

b

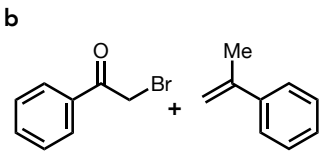

$11 a$

$12 \mathrm{a}$

Isotopically labelled Flavin

$D_{1}$-glucose, $\mathrm{H}_{2} \mathrm{O}$ buffer

Isotopically labelled buffer

$\mathrm{H}_{1}$-glucose, $\mathrm{D}_{2} \mathrm{O}$ buffer

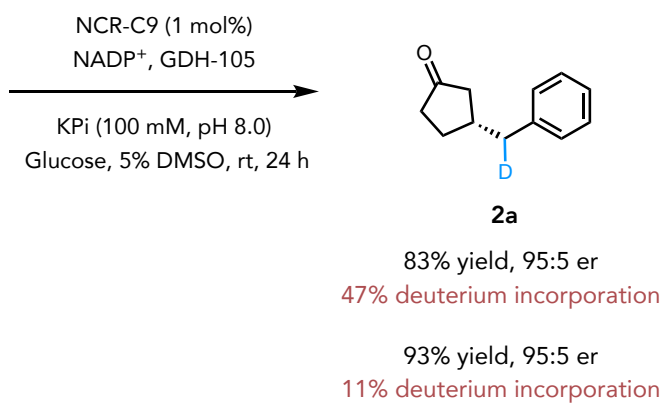

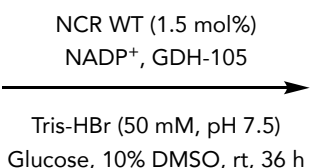

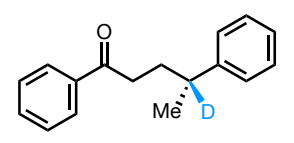

$13 a$

$42 \%$ yield, 99:1 er

$80 \%$ deuterium incorporation

$94 \%$ yield, 99:1 er

$<2 \%$ deuterium incorporation

Fig. 3. Deuterium labeling experiments. a. Deuterium labeling experiments for the cyclization mediated by NCR-C9. b. Deuterium labeling experiments for the intermolecular hydroalkylation catalyzed by wide-type NCR. Standard reaction conditions were used in all cases; for experimental details, see Supplementary Fig. 611.

\section{Conclusions}

In conclusion, we have discovered that the flavin-dependent enzyme NCR is capable of catalyzing $\mathrm{C}-\mathrm{C}$ bond-forming radical cyclization and that the activity and selectivity of the reaction can be significantly improved using directed evolution. The engineered 
quadruple mutant NCR-C9 shows broad substrate scope and excellent chemo- and stereoselectivity in the 5-exo-trig cyclization reaction, allowing the enantioselective synthesis of a variety of $\beta$-chiral cyclopentanones (Scheme 1). Moreover, we also developed the challenging asymmetric intermolecular radical $\mathrm{C}-\mathrm{C}$ bond-forming reactions using wild-type NCR as the biocatalyst, offering a valuable option for efficient preparation of $\gamma$-chiral ketones with high optical purity (Scheme 2). While the $\beta$-chiral center of cyclopentanone product was constructed by the $\mathrm{C}-\mathrm{C}$ bond-forming step in the cyclization catalyzed by NCR-C9, the $\gamma$-stereocenter of intermolecular hydroalkylation products produced by wild-type NCR was set via a stereoselective HAT process, thus demonstrating the versatile roles of enzymes with unparalleled capability in controlling stereoselectivity (Supplementary Fig. 5). Overall, our study showcases how non-natural reactivities of native EREDs can be exploited, which also can be further enhanced by protein engineering, if needed, to expand the biocatalyst toolbox and address the long-standing selectivity challenge in radical chemistry. We foresee EREDs and other flavin-dependent enzymes can be further leveraged to generate new synthetically applicable reactivities; this is particularly true as other fields, such as photocatalysis, ${ }^{20-22,35-37}$ are increasingly merging with biocatalysis. ${ }^{38,39}$ 


\section{Methods}

\section{Asymmetric radical cyclization reaction catalyzed by NCR-C9}

In the Coy chamber, a $5 \mathrm{~mL}$ vial was charged with GDH-105 $(100 \mu \mathrm{L}, 5 \mathrm{mg} / \mathrm{mL}$ stock solution in $100 \mathrm{mM} \mathrm{KPi}$ buffer $\mathrm{pH} 8.0)$, glucose (100 $\mu \mathrm{L}, 50 \mathrm{mg} / \mathrm{mL}$ stock solution), $\mathrm{NADP}^{+}(100 \mu \mathrm{L}, 5 \mathrm{mg} / \mathrm{mL}$ stock solution), NCR-C9 mutant (1.0 mol\%) and substrate 1a (25 $\mu \mathrm{L}, 160 \mathrm{mM}$ stock in DMSO, $0.004 \mathrm{mmol})$. Buffer (100 mM KPi buffer pH 8.0) was added to bring the total volume to $500 \mu \mathrm{L}$ with $5 \%$ DMSO $(v / v)$ as cosolvent. The vial was sealed with a screw cap and shaken under anaerobic conditions at room temperature for $24 \mathrm{~h}$. Upon completion, the reaction was quenched with $1.5 \mathrm{~mL}$ of acetonitrile and $50 \mu \mathrm{L}$ of $2 \mathrm{mg} / \mathrm{mL}$ 1,3,5-tribromobenzene (TBB) in acetonitrile as the internal standard. The mixture was shaken for $30 \mathrm{~min}$, centrifuged (12000 x g, 5 mins), and the supernatant was filtered and retained for LCMS analysis for yield calculation. After LCMS analysis, the supernatant was concentrated under reduced pressure, extracted with EtOAc. The combined organic layers were dried over $\mathrm{Na}_{2} \mathrm{SO}_{4}$, filtered, and concentrated under reduced pressure. The resulting crude residue was dissolved in $10 \%$ isopropanol/hexanes $(v / v)$ for chiral HPLC analysis.

\section{Wild-type NCR catalyzed radical intermolecular hydroalkylation}

In the Coy chamber, a $2 \mathrm{~mL}$ vial was charged with GDH-105 $(100 \mu \mathrm{L}, 3 \mathrm{mg} / \mathrm{mL}$ stock solution in $50 \mathrm{mM}$ Tris-HBr buffer $\mathrm{pH} 7.5)$, glucose (100 $\mu \mathrm{L}, 50 \mathrm{mg} / \mathrm{mL}$ stock solution), $\mathrm{NADP}^{+}(100 \mu \mathrm{L}, 2 \mathrm{mg} / \mathrm{mL}$ stock solution), NCR wild-type protein (1.5 mol\% to alkene), $\alpha$-bromoketone 11a (25 $\mu \mathrm{L}, 600 \mathrm{mM}$ DMSO stock solution, $0.015 \mathrm{mmol}, 3 \mathrm{eq})$ and alkene 12a (25 $\mu \mathrm{L}, 200 \mathrm{mM}$ stock in DMSO, $0.005 \mathrm{mmol}, 1 \mathrm{eq})$. Buffer (50 mM Tris$\mathrm{HBr} \mathrm{pH} 7.5$ ) and DMSO were added to bring the total volume to $1200 \mu \mathrm{L}$ with $10 \%$ $\operatorname{DMSO}(v / v)$. The vial was sealed with a screw cap and shaken under anaerobic conditions at room temperature for $36 \mathrm{~h}$. Upon completion, the reaction was quenched with $2.4 \mathrm{~mL}$ of acetonitrile and $50 \mu \mathrm{L}$ of $2 \mathrm{mg} / \mathrm{mL}$ 1,3,5-tribromobenzene (TBB) in acetonitrile as the internal standard. The mixture was shaken for $30 \mathrm{~min}$, centrifuged (12000 x g, 5 mins), and the supernatant was filtered and retained for LCMS analysis for yield calculation. After LCMS analysis, the supernatant was concentrated under 
reduced pressure, extracted with EtOAc. The combined organic layers were dried over $\mathrm{Na}_{2} \mathrm{SO}_{4}$, filtered, and concentrated under reduced pressure. The resulting crude residue was dissolved in $10 \%$ isopropanol/hexanes $(v / v)$ for chiral HPLC analysis.

\section{Detailed experimental procedures, chemical synthesis and analysis.}

Detailed experimental procedures and characterizations of compounds are provided in the Supplementary Information.

\section{Data availability}

Data are available in the Supplementary Information or from the corresponding author upon request. 


\section{References}

1. Plesniak, M. P., Huang, H.-M. \& Procter, D. J. Radical cascade reactions triggered by single electron transfer. Nat. Rev. Chem. 1, 1-16 (2017).

2. Yan, M., Lo, J. C., Edwards, J. T. \& Baran, P. S. Radicals: Reactive Intermediates with Translational Potential. Journal of the American Chemical Society 138, 12692-12714 (2016).

3. Studer, A. \& Curran, D. P. Catalysis of Radical Reactions: A Radical Chemistry Perspective. Angew. Chemie Int. Ed. 55, 58-102 (2016).

4. Ishibashi, H. Controlling the regiochemistry of radical cyclizations. Chem. Rec. 6, 23-31 (2006).

5. Liu, X., Ye, X., Bureš, F., Liu, H. \& Jiang, Z. Controllable Chemoselectivity in Visible-Light Photoredox Catalysis: Four Diverse Aerobic Radical Cascade Reactions. Angew. Chemie - Int. Ed. 54, 11443-11447 (2015).

6. Proctor, R. S. J., Colgan, A. C. \& Phipps, R. J. Exploiting attractive noncovalent interactions for the enantioselective catalysis of reactions involving radical intermediates. Nature Chemistry 12, 990-1004 (2020).

7. Sibi, M. P., Manyem, S. \& Zimmerman, J. Enantioselective radical processes. Chem. Rev. 103, 3263-3295 (2003).

8. Jäger, C. M. \& Croft, A. K. Anaerobic Radical Enzymes for Biotechnology. ChemBioEng Rev. 5, 143-162 (2018).

9. Tang, M. C., Zou, Y., Watanabe, K., Walsh, C. T. \& Tang, Y. Oxidative Cyclization in Natural Product Biosynthesis. Chemical Reviews 117, 52265333 (2017).

10. Walsh, C. T. \& Moore, B. S. Enzymatic Cascade Reactions in Biosynthesis. Angew. Chemie Int. Ed. 58, 6846-6879 (2019).

11. Nicolet, Y. Structure-function relationships of radical SAM enzymes. Nature Catalysis 3, 337-350 (2020).

12. Yokoyama, K. \& Lilla, E. A. C-C bond forming radical SAM enzymes involved in the construction of carbon skeletons of cofactors and natural products. Natural Product Reports 35, 660-694 (2018). 
13. Hyster, T. K. Synpacts Syn lett Radical Biocatalysis: Using Non-Natural Single Electron Transfer Mechanisms to Access New Enzymatic Functions. doi:10.1055/s-0037-1611818

14. Sandoval, B. A. \& Hyster, T. K. Emerging strategies for expanding the toolbox of enzymes in biocatalysis. Current Opinion in Chemical Biology 55, 45-51 (2020).

15. Toogood, H. S. \& Scrutton, N. S. Discovery, Characterization, Engineering, and Applications of Ene-Reductases for Industrial Biocatalysis. ACS Catalysis 8, 3532-3549 (2018).

16. Winkler, C. K., Faber, K. \& Hall, M. Biocatalytic reduction of activated $\mathrm{C}[\mathrm{dbnd}] \mathrm{C}-$ bonds and beyond: emerging trends. Current Opinion in Chemical Biology 43, 97-105 (2018).

17. Stewart\$, R. C. \& Massey, V. THE JOURNAL OF BIOLOGICAL CHEMISTRY Potentiometric Studies of Native and Flavin-substituted Old Yellow Enzyme*. 260, (1985).

18. Mansoorabadi, S. O., Thibodeaux, C. J. \& Liu, H. W. The diverse roles of flavin coenzymes - Nature's most versatile thespians. Journal of Organic Chemistry 72, 6329-6342 (2007).

19. Sandoval, B. A., Meichan, A. J. \& Hyster, T. K. Enantioselective Hydrogen Atom Transfer: Discovery of Catalytic Promiscuity in Flavin-Dependent 'Ene'Reductases. J. Am. Chem. Soc. 139, 11313-11316 (2017).

20. Biegasiewicz, K. F. et al. Photoexcitation of flavoenzymes enables a stereoselective radical cyclization. Science (80-. ). 364, 1166-1169 (2019).

21. Page, C. G. et al. Quaternary Charge-Transfer Complex Enables Photoenzymatic Intermolecular Hydroalkylation of Olefins. J. Am. Chem. Soc. (2020). doi:10.1021/jacs.0c11462

22. Huang, X. et al. Photoenzymatic enantioselective intermolecular radical hydroalkylation. Nature 584, 69-74 (2020).

23. Kirtland, S. J. Prostaglandin E1: A review. Prostaglandins, Leukotrienes and 
Essential Fatty Acids 32, 165-174 (1988).

24. Beale, M. H. \& Ward, J. L. Jasmonates: key players in the plant defence.

25. Gharpure, M. M. \& Rao, A. S. Total synthesis of (R)-(-)- $\beta$-cuparenone. Synth. Commun. 19, 679-688 (1989).

26. Westmeier, J., Kress, S., Pfaff, C. \& Von Zezschwitz, P. Total synthesis of (R)sarkomycin via asymmetric rhodium-catalyzed conjugate addition. J. Org. Chem. 78, 10718-10723 (2013).

27. Fäh, C. et al. New organofluorine building blocks: Inhibition of the malarial aspartic proteases plasmepsin II and IV by alicyclic $\alpha, \alpha$-difluoroketone hydrates. Org. Biomol. Chem. 7, 3947-3957 (2009).

28. Tanner, D. D. \& Singh, H. K. Reduction of $\alpha$-halo ketones by organotin hydrides. An electron-transfer-hydrogen atom abstraction mechanism. J. Org. Chem. 51, 5182-5186 (1986).

29. Jung, J., Kim, J., Park, G., You, Y. \& Cho, E. J. Selective Debromination and $\alpha$-Hydroxylation of $\alpha$-Bromo Ketones Using Hantzsch Esters as Photoreductants. Adv. Synth. Catal. 358, 74-80 (2016).

30. Tucker, J. W., Narayanam, J. M. R., Krabbe, S. W. \& Stephenson, C. R. J. Electron transfer photoredox catalysis: Intramolecular radical addition to indoles and pyrroles. Org. Lett. 12, 368-371 (2010).

31. Maji, T., Karmakar, A. \& Reiser, O. Visible-light photoredox catalysis: Dehalogenation of vicinal dibromo-, $\alpha$-halo-, and $\alpha, \alpha$-dibromocarbonyl compounds. J. Org. Chem. 76, 736-739 (2011).

32. Curran, D. P. \& Chang, C. tai. Atom Transfer Cyclization Reactions of $\alpha$-Iodo Esters, Ketones, and Malonates: Examples of Selective 5-Exo, 6-Endo, 6-Exo, and 7-Endo Ring Closures. J. Org. Chem. 54, 3140-3157 (1989).

33. Reich, S., Hoeffken, H. W., Rosche, B., Nestl, B. M. \& Hauer, B. Crystal Structure Determination and Mutagenesis Analysis of the Ene Reductase NCR. ChemBioChem 13, 2400-2407 (2012).

34. Sandoval, B. A., Kurtoic, S. I., Chung, M. M., Biegasiewicz, K. F. \& Hyster, T. 
K. Photoenzymatic Catalysis Enables Radical-Mediated Ketone Reduction in Ene-Reductases. Angew. Chemie Int. Ed. 58, 8714-8718 (2019).

35. Black, M. J. et al. Asymmetric redox-neutral radical cyclization catalysed by flavin-dependent 'ene'-reductases. Nat. Chem. 12, 71-75 (2020).

36. Clayman, P. D. \& Hyster, T. K. Photoenzymatic Generation of Unstabilized Alkyl Radicals: An Asymmetric Reductive Cyclization. J. Am. Chem. Soc. 142, $15673-15677(2020)$.

37. Sandoval, B. A. et al. Photoenzymatic Reductions Enabled by Direct Excitation of Flavin-Dependent “Ene”-Reductases. J. Am. Chem. Soc. (2020). doi:10.1021/jacs.0c11494

38. Rudroff, F. et al. Opportunities and challenges for combining chemo- and biocatalysis. Nature Catalysis 1, 12-22 (2018).

39. Schmermund, L. et al. Photo-Biocatalysis: Biotransformations in the Presence of Light. ACS Catalysis 9, 4115-4144 (2019).

\section{Acknowledgements}

This work was supported by the National Institutes of Health (NIH) National Institute of General Medical Sciences (NIGMS) (R01 GM127703) and the Princeton Catalysis Initiative. HL acknowledges the BioLEC Distinguished Postdoctoral Fellowship Program for support. The authors thank Dr. Yucong Zheng for his insightful discussions and assistance with docking. The authors also thank Josh Turek-Herman for generously providing the NCR single-site library, Jacob DeHovitz for sharing reference compounds, Jingzhe Cao for sharing intermediates, and Simon Charnock (Prozomix) for generously providing their ERED library. Dr. Ivan Keresztes, Anthony M. Condo, and Dr. John Eng are acknowledged for their help for measuring HRMS.

\section{Author contributions}

T.K.H conceived and directed the project. T.K.H, H.F., H.L., M.A.E., J.H.K. and B.A.S. designed the experiments. H.F., H.L., M.A.E., J.H.K. and B.A.S. performed and 
analyzed the results. T.K.H and H.F. wrote the manuscript with feedback from all other co-authors.

\section{Competing interests}

The authors declare no competing interests. 